\title{
Gravi-Weak Unification and the Black-Hole-Hedgehog's Solution with Magnetic Field Contribution
}

\author{
B.G. Sidharth ${ }^{1 *}$, C.R. Das ${ }^{2 \dagger}$ L.V. Laperashvili $\stackrel{3 \ddagger}{;}$ H.B. Nielsen ${ }^{4 \S}$ \\ ${ }^{1}$ International Institute of Applicable Mathematics \\ and Information Sciences, \\ B.M. Birla Science Centre \\ Adarsh Nagar, 500063 Hyderabad, India \\ ${ }^{2}$ Bogoliubov Laboratory of Theoretical Physics \\ Joint Institute for Nuclear Research \\ International Intergovernmental Organization, \\ Joliot-Curie 6, 141980 Dubna, Moscow region, Russia \\ 3 The Institute of Theoretical and Experimental Physics, \\ National Research Center "Kurchatov Institute", \\ Bolshaya Cheremushkinskaya, 25, 117218 Moscow, Russia \\ ${ }^{4}$ Niels Bohr Institute, \\ Blegdamsvej, 17-21, DK 2100 Copenhagen, Denmark
}

Keywords: black holes, hedgehogs, topological defects, effective potential, dark energy, cosmological constant, degenerate vacua

PACS: 04.50.Kd, 98.80.Cq, 12.10.-g, 95.35.+d, 95.36.+x

*iiamisbgs@yahoo.co.in, birlasc@gmail.com

†das@theor.jinr.ru

†laper@itep.ru

§hbech@nbi.dk 


\begin{abstract}
In the present paper, we investigated the gravitational black-hole-hedgehog's solution with magnetic field contribution in the framework of the $f(R)$-gravity described by the Gravi-Weak unification model. Assuming the Multiple Point Principle (MPP), we considered the existence of the two degenerate vacua of the Universe: the first Electroweak (EW) vacuum with $v_{1} \approx 246 \mathrm{GeV}$ ("true vacuum"), and the second Planck scale ("false vacuum") with $v_{2} \sim 10^{18} \mathrm{GeV}$. In these vacua, we investigated different topological defects. The main aim of this paper is an investigation of the black-hole-hedgehog configurations as defects of the "false vacuum". We have obtained the solution which corresponds to a global monopole, that has been "swallowed" by the black-hole with core mass $M_{B H} \approx 3.65 \times 10^{18} \mathrm{GeV}$ and radius $\delta \approx 6 \cdot 10^{-21} \mathrm{GeV}^{-1}$. We investigated the metric in the vicinity of the black-holehedgehog and estimated its horizon radius: $r_{h} \approx 1.14 \delta$. We have considered the phase transition from the "false vacuum" to the "true vacuum" and confirmed the stability of the EW-vacuum.
\end{abstract}

\title{
1 Introduction
}

In the previous papers, [1-3] devoted to studying of topological defects of the universal vacua we gave the investigation of hedgehog's configurations [4,5] neglecting the contribution of magnetic fields.

The birth of our Universe is a Big Bang since it represents the point of time when the Universe entered into a regime where the laws of physics began to work. Big Bang is not an explosion in space, but rather an expansion of space. After the initial expansion, the early Universe underwent a series of phase transitions. During these phase transitions, the breakdown of local or global gauge symmetries produces the vacuum topological defects (point, line and sheet defects). The cosmological model developed in Refs. [1 3] assumes the existence of two degenerate vacua of the Universe: The first ("true") Electroweak (EW) vacuum with VEV $v_{1} \approx 246 \mathrm{GeV}$, and the second ("false") Planck scale vacuum with VEV $v_{2} \sim 10^{18} \mathrm{GeV}$. In these papers, we investigated hedgehog's configurations as defects of "the false vacuum".

Gravitational black holes solutions with Yang-Mills fields were investigated explicitly in Ref. [6]. But in the present paper we investigate a solution for a global monopole, which is a black-hole-hedgehog at the Planck scale in the framework of the $f(R)$-gravity predicted by the Gravi-Weak unification (GWU) model, previously developed by authors in Refs. [7-10]. In contrast to the theory [1]3], here we have taken into account the contribution of the magnetic field of hedgehogs. 


\section{Gravi-Weak unification, the action and field equa- tions}

In Refs. [7-10], using results of Refs. [11, 12], we have constructed the Gravi-Weak unification (GWU), considering a Spin(4,4)-group of GWU spontaneously broken into the $S L(2, C)^{(\text {grav })} \times S U(2)^{(\text {weak })}$ group of symmetry. In agreement with experimental and astrophysical results, we assumed that after the Big Bang, there came into being the unification group $G_{T O E}$ of the Theory of the Everything (TOE) which was rapidly broken down to the direct product of series of gauge groups (see Ref. [1]) ended by the Standard Model group $G_{S M}$ :

$$
G S M=S U(3)_{c} \times S U(2)_{L} \times U(1)_{Y}
$$

The action $S_{(G W)}$ of the Gravi-Weak unification obtained in Refs. [7-10] is given by the following expression:

$$
\begin{aligned}
S_{(G W)}= & -\frac{1}{g_{\text {uni }}} \int_{\mathfrak{M}} d^{4} x \sqrt{-g}\left[\frac{1}{16}\left(R|\Phi|^{2}-\frac{3}{2}|\Phi|^{4}\right)\right. \\
& \left.+\frac{1}{16}\left(a R_{\mu \nu} R^{\mu \nu}+b R^{2}\right)+\frac{1}{2} \mathcal{D}_{\mu} \Phi^{\dagger} \mathcal{D}^{\mu} \Phi+\frac{1}{4} F_{\mu \nu}^{i} F^{i \mu \nu}\right],
\end{aligned}
$$

where $g_{\text {uni }}$ is a parameter of the GWU, parameters $a, b$ (with $a+b=1$ ) are "bare" coupling constants of the higher derivative gravity, $R$ is the Riemann curvature scalar, $R_{\mu \nu}$ is the Ricci tensor, $|\Phi|^{2}=\Phi^{a} \Phi^{a}$ is a squared triplet Higgs field, where $\Phi^{a}$ (with $a=1,2,3$ ) is an isovector scalar belonging to the adjoint representation of the $S U(2)$ gauge group of symmetry. In Eq.(2):

$$
\mathcal{D}_{\mu} \Phi^{a}=\partial_{\mu} \Phi^{a}+g_{2} \epsilon^{a b c} A_{\mu}^{b} \Phi^{c}
$$

is a covariant derivative, and

$$
F_{\mu \nu}^{a}=\partial_{\mu} A_{\nu}^{a}-\partial_{\nu} A_{\mu}^{a}+g_{2} \epsilon^{a b c} A_{\mu}^{b} A_{\nu}^{c}
$$

is a curvature of the gauge field $A_{\mu}^{a}$ of the $S U(2)$ Yang-Mills theory with a coupling constant $g_{2}$ as a "bare" coupling constant of the $S U(2)$ weak interaction.

The action (2) is a special case of the $f(R)$ gravity [13-15] when:

$$
f(R)=R|\Phi|^{2} .
$$

General case of the $f(R)$ gravity gives the action containing matter fields and can be presented by the following expression:

$$
S=\frac{1}{2 \kappa} \int d^{4} x \sqrt{-g} f(R)+S_{\text {grav }}+S_{\text {gauge }}+S_{m},
$$

where the action $S_{m}$ is associated with matter fields (fermions and Higgs fields).

From the action (2), using the metric formalism, we obtain the following field equations:

$$
\mathcal{F}(R) R_{\mu \nu}-\frac{1}{2} f(R) g_{\mu \nu}-\nabla_{\mu} \nabla_{\nu} \mathcal{F}(R)+g_{\mu \nu} \square \mathcal{F}(R)=\kappa T_{\mu \nu}^{m},
$$


where:

$$
\left.\mathcal{F}(R) \equiv \frac{d f(r)}{d r}\right|_{r=R}
$$

$\kappa=8 \pi G_{N}, G_{N}$ is the gravitational constant, and $T^{m}$ is the energy-momentum tensor derived from the matter action $S_{m}$.

Varying the fields $\Phi$ and $A_{\mu}$, we obtain the next field equations:

$$
\mathcal{D}_{\mu} \mathcal{D}^{\mu} \Phi=\left(\frac{R}{8}-\lambda|\Phi|^{2}\right) \Phi
$$

where according to Ref [1] (see Appendix A, Eq. (A13)), we have:

$$
\lambda=\frac{3 g_{2}^{2}}{8}
$$

and

$$
\mathcal{D}^{\mu} F_{\mu \nu}=-J_{\nu}
$$

where $J_{\nu}$ is a current, produced by the Higgs field $\Phi^{a}$ :

$$
J_{\nu}=\frac{1}{2}\left[\Phi^{\dagger} \mathcal{D}_{\nu} \Phi-\left(\mathcal{D}_{\nu} \Phi^{\dagger}\right) \Phi\right]
$$

\section{De Sitter solutions at the early time of the Uni- verse}

It is well known that at the early time, the Universe is described by the de Sitter solutions (see for example Refs. [16, 17]). Our model is a special case of the more general SU(N) model [11], where authors assumed that the Universe is inherently de Sitter. Then, the 4-spacetime is a hyperboloid in a 5-dimensional Minkowski space under the constraint:

$$
x_{0}^{2}+x_{1}^{2}+x_{2}^{2}+x_{3}^{2}+x_{4}^{2}=r_{d S}^{2},
$$

where $r_{d S}$ is a radius of the curvature of the de Sitter space, or simply "the de Sitter radius".

Vacuum energy density of our Universe is the Dark Energy (DE). The cosmological constant $\Lambda$ describes the DE substance, which is dominant in the Universe at later times:

$$
\Omega_{D E}=\frac{\rho_{D E}}{\rho_{\text {crit }}} \simeq 0.75,
$$

where $\rho_{D E}$ is the dark energy density and the critical density is:

$$
\rho_{\text {crit }}=\frac{3 H_{0}^{2}}{8 \pi G_{N}} \simeq 1.88 \times 10^{-29} H_{0}^{2} .
$$

Here $H_{0}$ is the Hubble constant:

$$
H_{0} \simeq 1.5 \times 10^{-42} \mathrm{GeV} .
$$


Dark Energy (DE) is related with cosmological constant $\Lambda$ by the following way:

$$
\rho_{D E}=\rho_{v a c}=\left(M_{P l}^{r e d}\right)^{2} \Lambda
$$

where $M_{P l}^{r e d}$ is the reduced Planck mass: $M_{P l}^{r e d} \simeq 2.43 \times 10^{18} \mathrm{GeV}$.

At present, cosmological measurements give (see [18]):

$$
\rho_{D E} \simeq\left(2 \times 10^{-3} \mathrm{eV}\right)^{4}
$$

which means a tiny value of the cosmological constant:

$$
\Lambda \simeq 10^{-84} \mathrm{GeV}^{2}
$$

This tiny value of $\rho_{D E}$ was first predicted by B.G. Sidharth in 1997 year [19,20]. In the 1998 year S. Perlmutter, B. Schmidt and A. Riess [21] were awarded the Nobel Prize for the discovery of the accelerating expansion of the Universe.

Having an extremely small cosmological constant of our Universe, Bennett, Froggatt and Nielsen [22 24] assumed to consider only zero, or almost zero, cosmological constants for all vacua existing in Nature. They formulated a new law of Nature named the Multiple Point Principle (MPP), which means: There exist in Nature several degenerate vacua with very small energy density, or cosmological constants.

The model developed in this article considers the existence of the two degenerate vacua of the Universe: The first ("true") Electroweak (EW) vacuum, and the second ("false") Planck scale vacuum.

From experimental results, cosmological constants - minima of the Higgs effective potentials $V_{\text {eff }}\left(\phi_{H}\right)$ - are not exactly equal to zero. Nevertheless, they are extremely small. By this reason, Bennett, Froggatt and Nielsen [22-24] assumed to consider zero cosmological constants as a good approximation. Then according to the MPP, we have a model of pure SM being fine-tuned in such a way that these two vacua proposed have just zero energy density (see also Ref. [25]).

If the effective potential has two degenerate minima, then the following requirements are satisfied:

$$
V_{\text {eff }}\left(\phi_{\min 1}^{2}\right)=V_{\text {eff }}\left(\phi_{\min 2}^{2}\right)=0
$$

and

$$
V_{e f f}^{\prime}\left(\phi_{\min 1}^{2}\right)=V_{e f f}^{\prime}\left(\phi_{\min 2}^{2}\right)=0
$$

where

$$
V^{\prime}\left(\phi^{2}\right)=\frac{\partial V}{\partial \phi^{2}}
$$

Here we assume that:

$$
V_{\text {eff }}\left(\phi_{\min 1}^{2}\right)=V_{E W}, \quad V_{\text {eff }}\left(\phi_{\min 2}^{2}\right)=V_{\text {high field }} .
$$

Assuming the existence of the two degenerate vacua in the SM:

a) the first Electroweak vacuum at $v_{1} \approx 246 \mathrm{GeV}$, and 
b) the second Planck scale vacuum at $v_{2} \sim 10^{18} \mathrm{GeV}$, Froggatt and Nielsen predicted in Ref. 24] the top-quark and Higgs boson masses:

$$
M_{t}=173 \pm 5 \mathrm{GeV} ; \quad M_{H}=135 \pm 10 \mathrm{GeV} .
$$

In the present paper we study the evolution of the Universe as two bubbles: one having a "false vacuum", and the other one having a "true vacuum". The bubble, which we shall refer to as the false vacuum, to be a de Sitter space with a constant expansion rate $H_{F}$. This bubble has a radius close to the de Sitter horizon, which corresponds to the Universe radius:

$$
R_{\text {un }} \simeq R_{\text {de Sitter horizon }} \simeq 10^{28} \mathrm{~cm} .
$$

It is convenient to use the flat de Sitter coordinates to describe the background of the inflating false vacuum:

$$
d s^{2}=d t^{2}-e^{2 H_{F} t}\left(d r^{2}+r^{2} d \Omega^{2}\right),
$$

where

$$
d \Omega^{2}=d \theta^{2}+\sin ^{2} \theta d \phi^{2} .
$$

The space-time inside the bubble, which we shall refer to as a true vacuum, has the geometry of an open Friedmann-Lemaitre-Robertson-Walker (FLRW) universe (see for example review [26]):

$$
d s^{2}=d \tau^{2}-a(\tau)^{2}\left(d \xi^{2}+\sinh ^{2} \xi d \Omega^{2}\right),
$$

where $a(\tau)$ is a scale factor with cosmic time $\tau$. In the true vacuum we have a constant expansion rate $H_{T}$, which has the meaning of the slow-roll inflation rate inside the bubble at the early stage of its evolution.

Cosmological theory of bubbles was developed in a lot of papers by A. Vilenkin and his collaborators (see for example, Refs. [27-30]).

As it was shown in Ref. [11], the nontrivial vacuum solution to the action (2) is de Sitter spacetime with a non-vanishing Higgs vacuum expectation value (VEV) of the triplet Higgs scalar field $\Phi: v_{2}=\langle\Phi\rangle=\Phi_{0}$. The standard Higgs potential in Eq.(2) has an extremum at $\Phi_{0}=R / 3$ (with $R>0$ ), corresponding to a de Sitter spacetime background solution:

$$
R=R_{0}=\frac{12}{r_{d S}^{2}}=3 v_{2}^{2}
$$

which implies vanishing curvature:

$$
F_{0}=\frac{1}{2} R_{0}-\frac{1}{16} \Sigma_{0} \Phi_{0}^{2}
$$

solving the field equations $D F=d F+[A, F]=0$, and strictly minimizing the action (2).

Based on this picture, the origin of the cosmological constant (and DE) is associated with the inherent spacetime geometry, and not with vacuum energy of particles (we consider their contributions later). We note that as a fundamental constant under the de Sitter symmetry, $r_{d S}$ is not a subject to quantum corrections. Local dynamics exist as fluctuations with respect to this cosmological background. In general, the de Sitter space may be inherently unstable. The quantum instability of the de Sitter space was investigated by various authors. Abbott and Deser [31] have shown that de Sitter space is stable under a restricted class of classical gravitational perturbations. So any instability 
of the de Sitter space may likely have a quantum origin. Ref. [32] demonstrated through the expectation value of the energy-momentum tensor for a system with a quantum field in a de Sitter background space, that in general, it contains a term that is proportional to the metric tensor and grows in time. As a result, the curvature of the spacetime would decrease and the de Sitter space tends to decay into the flat space (see Ref. [33]). The decay time of this process is of the order of the de Sitter radius:

$$
\tau \sim r_{d S} \simeq 1.33 H_{0}^{-1}
$$

Since the age of our universe is smaller than $\tau_{d S}$, we are still observing the accelerating expansion of the Universe.

Of course, we also can consider the perturbation de Sitter solutions but these perturbations are very small [16, 17].

\section{The solution for the gravitational black-holes-hedgehogs with magnetic field contribution}

The field configurations describing a monopole-hedgehog [4,5] are:

$$
\begin{gathered}
\Phi^{a}=v w(r) \frac{x^{a}}{r}, \\
A_{\mu}^{a}=a(r) \epsilon_{\mu a b} \frac{x^{b}}{r},
\end{gathered}
$$

where $x^{a} x^{a}=r^{2}$ with $(a=1,2,3), w(r)$ and $a(r)$ are some structural functions. This solution is pointing radially. Here $\Phi^{a}$ is parallel to $\hat{r}$ - the unit vector in the radial, and we have a "hedgehog" solution of Refs. [4, 5]. The terminology "hedgehog" was first suggested by Alexander Polyakov in Ref. [5].

In the flat metric, the field equations (9) for $\Phi^{a}$ give the following equation for $w(r)$ :

$$
w^{\prime \prime}+\frac{2}{r} w^{\prime}-\frac{2}{r^{2}} w-\frac{w\left(w^{2}-1\right)}{\delta^{2}}-2 w a^{2}(r)=0,
$$

where $\delta$ is a core radius of the hedgehog.

The function $w(r)$ grows with $r$ from $w(0)=0$ and exponentially approaches to the unity: $\lim w(r)_{r \rightarrow \infty}=1$. Barriola and Vilenkin [27] took $w=1$ outside the core when $r \geq \delta$, which is an approximation to the exact solution. As a result, the functions $w(r)$ and $a(r)$ are constrained by the following conditions:

$$
\begin{gathered}
w(0)=0, \quad \text { and } \quad w(r) \rightarrow 1 \quad \text { when } \quad r \rightarrow \infty, \\
a(0)=0, \quad \text { and } \quad a(r) \sim-\frac{g}{r} \quad \text { when } \quad r \rightarrow \infty .
\end{gathered}
$$




\subsection{The metric in the vicinity of the global monopole}

The most general static metric in the vicinity of the global monopole is a metric with a spherical symmetry:

$$
d s^{2}=B(r) d t^{2}-A(r) d r^{2}-r^{2}\left(d \theta^{2}+\sin ^{2} \theta d \varphi^{2}\right) .
$$

For this metric the Ricci tensor has the following non-vanishing components:

$$
\begin{gathered}
R_{t t}=-\frac{B^{\prime \prime}}{2 A}+\frac{B^{\prime}}{4 A}\left(\frac{A^{\prime}}{A}+\frac{B^{\prime}}{B}\right)-\frac{1}{r} \frac{B^{\prime}}{A}, \\
R_{r r}=\frac{B^{\prime \prime}}{2 B}+\frac{B^{\prime}}{4 B}\left(\frac{A^{\prime}}{A}+\frac{B^{\prime}}{B}\right)-\frac{1}{r} \frac{A^{\prime}}{A}, \\
R_{\theta \theta}=-1+\frac{r}{2 A}\left(-\frac{A^{\prime}}{A}+\frac{B^{\prime}}{B}\right)+\frac{1}{A}, \\
R_{\varphi \varphi}=\sin ^{2} \theta R_{\theta \theta} .
\end{gathered}
$$

Now we can calculate the global monopole energy-momentum tensor components:

$$
\begin{gathered}
T_{t}^{t}=v^{2} \frac{w^{\prime 2}}{2 A}+v^{2} \frac{w^{2}}{r^{2}}+\frac{1}{4} \lambda v^{4}\left(w^{2}-1\right)^{2}-\frac{a^{\prime 2}}{A}+\frac{a^{2}}{r^{2}} \\
T_{r}^{r}=-v^{2} \frac{w^{\prime 2}}{2 A}+v^{2} \frac{w^{2}}{r^{2}}+\frac{1}{4} \lambda v^{4}\left(w^{2}-1\right)^{2}-\frac{a^{\prime 2}}{A}+\frac{a^{2}}{r^{2}} \\
T_{\theta}^{\theta}=T_{\varphi}^{\varphi}=v^{2} \frac{w^{\prime 2}}{2 A}+\frac{1}{4} \lambda v^{4}\left(w^{2}-1\right)^{2} .
\end{gathered}
$$

Here $\kappa=1$.

\subsection{The hedgehog's structure functions}

As an example we can use the following expressions for monopole structure functions $w(r)$ and $a(r)$, which satisfy the conditions (34) and (35):

$$
\begin{gathered}
w(r)=1-\exp \left(-\frac{r^{2}}{\delta^{2}}\right), \\
a(r)=-\frac{g}{r}\left(1-\exp \left(-\frac{r^{2}}{\delta^{2}}\right)\right),
\end{gathered}
$$

Then we see that in the vicinity of $r \rightarrow 0$ we have:

$$
w(r)=\frac{r^{2}}{\delta^{2}}+\ldots \quad \text { for } \quad r \rightarrow 0
$$

and

$$
a(r)=-g \frac{r}{\delta^{2}}+\ldots \quad \text { for } \quad r \rightarrow 0,
$$

But in the vicinity of $r \rightarrow \infty$ we have:

$$
w(r) \rightarrow 1-\ldots \text { for } r \rightarrow \infty,
$$


and

$$
a(r) \rightarrow-\frac{g}{r}+\ldots \text { for } r \rightarrow \infty
$$

in accordance with the conditions (34) and (35).

Of course, we are able to calculate the components (38) of the monopole energymomentum tensor using the result (39) and (40). But for simple estimation we can be limited by an approximation:

$$
w(r)=0 \quad \text { for } \quad 0 \leq r \leq \delta, \quad \text { and } \quad w(r)=1 \quad \text { for } \quad \delta<r<\infty
$$

and

$$
a(r)=0, \quad g=0 \quad \text { for } \quad 0 \leq r \leq \delta, \quad \text { and } \quad a(r)=-\frac{g}{r}, \quad g=g_{2} \quad \text { for } \quad \delta<r<\infty,
$$

where $\delta$ is a radius of the hedgehog.

\subsection{The solution with magnetic field contribution}

Considering the approximation (45) and (46), in agreement with a solution used by Barriola and Vilenkin in Ref. [27], we can obtain a simple approximate solution for the monopole-hedgehog taking $w=1$ out the core of the hedgehog [1]. In the case of Refs. [1, 36 40] scalar curvature $R$ is constant, and Eq. (7) comes down to the Einstein's equation:

$$
\begin{aligned}
& \frac{1}{A}\left(\frac{1}{r^{2}}-\frac{1}{r} \frac{A^{\prime}}{A}\right)-\frac{1}{r^{2}}=\frac{1}{\kappa v^{2}} T_{t}^{t}, \\
& \frac{1}{A}\left(\frac{1}{r^{2}}+\frac{1}{r} \frac{B^{\prime}}{B}\right)-\frac{1}{r^{2}}=\frac{1}{\kappa v^{2}} T_{r}^{r} .
\end{aligned}
$$

Here $\kappa=8 \pi G_{N}$.

In approximation (45) and (46), the energy-momentum tensor components are given by the following approximations:

$$
\begin{gathered}
T_{t}^{t}=T_{r}^{r} \approx \frac{\lambda \kappa^{2} v^{4}}{4} \text { for } 0 \leq r \leq \delta, \\
T_{t}^{t}=T_{r}^{r} \approx \frac{\kappa v^{2}}{r^{2}}+\frac{g^{2}}{r^{4}}\left(-\frac{1}{A}+1\right) \quad \text { for } \quad \delta<r<\infty,
\end{gathered}
$$

By substraction of Eqs.(47) and (48) we obtain:

$$
\frac{A^{\prime}}{A}+\frac{B^{\prime}}{B}=0
$$

From Eq. (47) we obtain a general relation for the function $A(r)$ :

$$
A^{-1}(r)=1-\frac{1}{r} \int_{0}^{r} T_{t}^{t} r^{2} d r .
$$

Using expressions (49) and (50), we obtain:

$$
A^{-1}(r)=1-\frac{\lambda \kappa^{2} v^{4}}{4 r} \int_{0}^{\delta} r^{2} d r-\frac{1}{r} \int_{\delta}^{r}\left(\frac{\kappa v^{2}}{r^{2}}+\frac{g^{2}}{r^{4}}\left(-\frac{1}{A}+1\right)\right) r^{2} d r .
$$


Taking into account the Schwarzschild type metric for a black-hole given by Refs. [1,6, 41], we can use the following expression for $A^{-1}(r)$ :

$$
A^{-1}(r) \approx C+\frac{C_{1}}{r}+\frac{C_{2}}{r^{2}}+\frac{C_{3}}{r^{3}}+\ldots,
$$

and from Eq. (53) we calculate:

$$
C=1-\kappa v^{2}, \quad C_{1}=\kappa v^{2} \delta, \quad C_{2}=g^{2} \kappa v^{2}, \quad C_{3}=-\frac{g^{2}}{2} \kappa v^{2} \delta, \ldots
$$

Eq. (51) gives:

$$
A(r)=B^{-1}(r),
$$

and finally we obtain the following result:

$$
A^{-1}(r)=B(r) \approx 1-\kappa v^{2}+\frac{\kappa v^{2} \delta}{r}+\frac{g^{2} \kappa v^{2}}{r^{2}}-\frac{g^{2} \kappa v^{2} \delta}{2 r^{3}}+\ldots
$$

According to Refs. [1,6, 41], the Schwarzschild type metric for a black-hole is given by the expression:

$$
A^{-1} \approx 1-\kappa v^{2}-\frac{2 G_{N} M}{r}+\ldots,
$$

where $M$ is a black-hole's mass parameter, which in our theory is given by the following expression:

$$
M \approx-4 \pi v^{2} \delta
$$

The mass parameter (59) is negative. There is a repulsive gravitational potential due to this negative mass parameter given by the metric parameter $A(r)$. But this parameter $M$ is not a mass of the hedgehog: the black-hole-hedgehog has a positive mass:

$$
M_{B H}=-M=4 \pi v^{2} \delta .
$$

If we take the space integral of the hedgehog energy density as given by (49, 50), say - this would be total energy of the hedgehog in the ignoring gravity approximation - the integration over the radius $r$ will diverge for large $r$, because of the term $\frac{\kappa v^{2}}{r^{2}}$ in (50). So with the approximations done the a priori "mass" = "energy" of the hedgehog is $+\infty$. The positivity is what one expects for a disturbance in a background vacuum, which has minimum energy density and the divergence comes from the kinetic term due to the variation of the $\Phi^{a}$ field because of having different directions in a component space essentially following the direction in space from the center out. Indeed such a variation leads to a gradient square term behaving $\propto \frac{1}{r^{2}}$. When this is integrated over space - meaning an integral $\int \ldots 4 \pi r^{2} d r$ one gets a term proportional to upper end $r$ and thus divergence. It is a kind of infrared divergence in the sense that it comes from large distance scales.

This is a priori looking like a hedgehog-"soliton" having an infinite energy or mass. And it is needed to give some comments on it and its understanding:

- In fact, this divergence causing contribution is not really there if we calculate fully in our Gravi-Weak theory in as far as the contribution is showing up as just a gauge artefact if we include the gauge field associated with the transformation of 
components of the $\Phi^{a}$ scalar field. As one sees from the Lagrangian the kinetic term for the $\Phi^{a}$ field involves covariant derivatives, and then one can arrange a lower energy density by letting the Yang-Mills fields adjust to make the actual values of these covariant derivatives in the far-out surroundings of the hedgehog be zero. As a price for this vanishing of the covariant derivatives one has to accept the magnetic fields as they then come, but this gives crudely more differentiation and a dependence with a more negative power of $r$ ensuring convergence in the large $r$ limit.

So really this problem of divergence - if we consider it a problem - is due to having ignored the Yang-Mills field.

- As one may note from the performed calculation the divergent causing term goes into the r-independent term $C$ in the expansion (154) of the inverse of $A$, rather than into the term going as $C_{1} / r$ as one would expect for a mass. One could look at this phenomenon as the constant coefficient $C$ in the $A^{-1}$ expansion somehow "renormalizing" the mass so as to take up in itself the divergent part.

- It is only because of there being such "renormalization-like" trick going on in the calculation that it can at all be possible to circumvent the a priori expectation that "the mass $M$ " must, of course, be positive because of the hedgehog being an excitation on the background of a ground state.

But once one can interpret part of the a priori mass contribution as something else by shuffling it into the constant $C$ term in the (54) expansion, of course even the sign of the rest - which then is interpreted indeed as a mass $M$ - gets out of control and there is no contradiction by it being negative.

\section{Lattice-like structure of the false vacuum}

Now we can construct the lattice-like topological contribution with negative vacuum energy density.

Assuming that black-holes with mass parameter $M=-M_{B H}$ form a hypercubic lattice with lattice parameter $l=\lambda_{P l}$, we have the negative energy density (and negative cosmological constant $\Lambda_{\text {lat }}$ ) of such a lattice equal to:

$$
\rho_{\text {lat }} \simeq-M_{B H} M_{P l}^{3}=\Lambda_{l a t} M_{P l}^{2} .
$$

If this energy density of the hedgehogs lattice compensates the Einstein's vacuum energy (see (A10) and (A15)), we have the following equation:

$$
\frac{\lambda}{4} v^{4} \approx M_{B H} M_{P l}^{3}
$$

Using the estimation (A8), we obtain:

$$
\frac{3}{2} M_{P l}^{4} \approx M_{B H} M_{P l}^{3}
$$

or

$$
M_{B H}=\frac{3}{2} M_{P l} \approx 3.65 \times 10^{18} \mathrm{GeV}
$$


Therefore black-holes-hedgehogs have a huge mass of order of the Planck mass.

Eq.(60) predicts a radius $\delta$ of the hedgehog's core:

$$
\delta \approx \frac{M_{B H}}{4 \pi v^{2}} \approx\left(\frac{64 \pi}{3} M_{P l}\right)^{-1} \approx 6 \cdot 10^{-21} \mathrm{GeV}^{-1}
$$

\subsection{The hedgehog's horizon radius}

We have obtained a global monopole with a huge mass (64). This is a black-hole solution, which corresponds to a global monopole-hedgehog that has been "swallowed" by a blackhole. Indeed, we have obtained the metric result by M. Barriola et al. [27] like:

$d s^{2}=\left(1-\kappa v^{2}+\frac{2 G_{N} M_{B H}}{r}+\ldots\right) d t^{2}-\frac{d r^{2}}{\left(1-\kappa v^{2}+\frac{2 G_{N} M_{B H}}{r}+\ldots\right)}-r^{2}\left(d \theta^{2}+\sin ^{2} \theta d \varphi^{2}\right)$.

A black hole has a horizon. A horizon radius $r_{h}$ can be found by solving the equation:

$$
A^{-1}\left(r_{h}\right)=0
$$

Assuming that $g^{2}=0$ in the region $r \leq r_{h}$, we obtain:

$$
\left(1-\kappa v^{2}\right)\left(1+\frac{2 G_{N} M_{B H}}{\left(1-\kappa v^{2}\right) r_{h}}\right)=0 .
$$

Eq. (68) gives a solution for hedgehog's horizon radius:

$$
r_{h} \approx \frac{\kappa M_{B H}}{4 \pi\left(\kappa v^{2}-1\right)}
$$

According to Eq. (A7), $\kappa v^{2}=8$, and the black-hole-hedgehog's horizon radius is equal to:

$$
r_{h} \approx \frac{\frac{\kappa}{4 \pi} \times M_{B H}}{1-\kappa v^{2}}=\frac{\kappa v^{2} \delta}{1-\kappa v^{2}} \approx \frac{8}{7} \delta \approx 1.14 \delta .
$$

We see that the horizon radius $r_{h}$ is larger than the hedgehog radius $\delta$ :

$$
r_{h}>\delta
$$

and our concept that "a black hole contains the hedgehog" is justified.

\subsection{Lattice-like structure of the false vacuum and non-commutativity}

We see that at the Planck scale the false vacuum of the Universe is described by a nondifferentiable space-time: by a foam of black-holes, having lattice-like structure, in which sites are black-holes with "hedgehog" monopoles inside them. This manifold is described by a non-commutative geometry (see Ref. [1]).

In Refs. [19,20] B.G. Sidharth predicted: 
1. That a cosmological constant is given by a tiny value:

$$
\Lambda \sim H_{0}^{2}
$$

where $H_{0}$ is the Hubble rate in the early Universe:

$$
H_{0} \simeq 1.5 \times 10^{-42} \mathrm{GeV}
$$

2. That a Dark Energy density is very small:

$$
\rho_{D E} \simeq 10^{-12} \mathrm{eV}^{4}=10^{-48} \mathrm{GeV}^{4}
$$

3. That a very small DE-density provides an accelerating expansion of our Universe after the Big Bang.

Sidharth proceeded from the following points of view [42]: Modern Quantum Gravity [43] (Loop Quantum Gravity, etc.,) deal with a non-differentiable space-time manifold. In such an approach, there exists a minimal space-time cut off $\lambda_{\min }$, which leads to the non-commutative geometry.

If the space-time is fuzzy, non-differentiable, then it has to be described by a noncommutative geometry with the coordinates obeying the following commutation relations:

$$
\left[d x^{\mu}, d x^{\nu}\right] \approx \beta^{\mu \nu} l^{2} \neq 0
$$

Eq. (74) is true for any minimal cut off $l$.

Previously the following commutation relation was considered by H.S. Snyder [44]:

$$
[x, p]=\hbar\left(1+\left(\frac{l}{\hbar}\right)^{2} p^{2}\right), \text { etc. }
$$

which shows that effectively 4 -momentum $p$ is replaced by

$$
p \rightarrow p\left(1+\left(\frac{l}{\hbar}\right)^{2} p^{2}\right)^{-1}
$$

Then the energy-momentum formula becomes as:

$$
E^{2} \approx m^{2}+p^{2}-2\left(\frac{l}{\hbar}\right)^{2} p^{4} .
$$

In such a theory the usual energy momentum dispersion relations are modified. In the above equations, $l$ stands for a minimal (fundamental) length, which could be the Planck length $\lambda_{P l}$, or for more generally - Compton wavelength $\lambda_{c}$.

Writing Eq. (77) as

$$
E=E^{\prime}+E^{\prime \prime}
$$

where $E^{\prime}$ is the usual (old) expression for energy, and $E^{\prime \prime}$ is the new additional term in modification. $E^{\prime \prime}$ can be easily verified as $E^{\prime \prime}=-m_{b} c^{2}$ for boson fields, and $E^{\prime \prime}=+m_{f} c^{2}$ 
- for fermion fields with masses $m_{b}, m_{f}$, respectively. These formulas help to identify the DE density, what was first realized by B.G. Sidharth in Ref. [20].

DE density is a density of the quantum vacuum energy of the Universe. Quantum vacuum, described by Zero Point Fields (ZPF) contributions is the lowest state of any Quantum Field Theory (QFT), and due to the Heisenberg's principle has an infinite value, which is renormalizable.

As it was pointed out in Refs. [45, 46], the quantum vacuum of the Universe can be a source of the cosmic repulsion. However, a difficulty in this approach has been that the value of the cosmological constant turns out to be huge [46], far beyond the value which is observed by astrophysical measurements. This phenomenon has been called "the cosmological constant problem" [47].

A global monopole is a heavy object formed as a result of the gauge-symmetry breaking during the phase transition of the isoscalar triplet $\Phi^{a}$ system. The black-holeshedgehogs are similar to elementary particles, because of a major part of their energy is concentrated in a small region near the monopole core. Assuming that the Planck scale false vacuum is described by a non- differentiable space-time having lattice-like structure, where sites of the lattice are black-holes with "hedgehog" monopoles inside them, we describe this manifold by a non-commutative geometry with a minimal length $l=\lambda_{P l}$.

The result (61) is in agreement with the result of the non-commutativity (78) with $E^{\prime \prime}=-M_{B H} c^{2}$, because black-holes-hedgehogs are point-like topological defects similar to scalar particles giving the negative contribution $\rho_{\text {lat }}<0$ to the vacuum energy density $\rho_{D E}$ of the Universe.

Using the non-commutative theory of the discrete space-time, B.G. Sidharth predicted in Refs. [20,45] a tiny value of the cosmological constant: $\Lambda \simeq 10^{-84} \mathrm{GeV}^{2}$ as a result of the compensation of ZPF contributions by non-commutative contributions of the (boson and fermion) lattices.

\section{The phase transition from the "false vacuum" to the "true vacuum"}

In the present model, we investigated the evolution of the two bubbles of the Universe, considering two phases of the universal vacua:

1. one being a "false vacuum" (Planck scale vacuum), and

2. the other is a "true vacuum" (EW-vacuum).

The cosmological model predicts that the Universe exists in the Planck scale phase for extremely short time. For this reason, the Planck scale phase was called "the false vacuum". The presence of hedgehogs as vacuum defects is responsible for the destabilization of the false vacuum. The decay of the false vacuum is accompanied by the decay of the black-holes-hedgehogs. These configurations are unstable, and at some finite cosmic 
temperature which is called the critical temperature $T_{c}$, a system exhibits a spontaneous symmetry breakdown, and we observe a phase transition from the bubble with the false vacuum to the bubble with the true vacuum. After the phase transition, the Universe begins its evolution toward the low energy Electroweak (EW) phase. Here the Universe underwent the inflation, which led to the phase having the VEV $v_{1} \approx 246 \mathrm{GeV}$. This is a "true" vacuum, in which we live.

Ref. [36] also allowed a possibility to consider an arbitrary domain wall between these two phases. During the inflation, domain wall annihilates, producing gravitational waves and a lot of the SM particles, having masses.

The Electroweak spontaneous breakdown of symmetry $S U(2)_{L} \times U(1)_{Y} \rightarrow U(1)_{\text {el.mag }}$ leads to the creation of the topological defects of the EW-vacuum. They are the AbrikosovNielsen-Olesen closed magnetic vortices ("ANO strings") of the Abelian Higgs model [48, 49], and Sidharth's Compton phase objects [50, 51]. Then the Electroweak vacuum and high-field "false vacuum" both present the non-differentiable manifold, described by the non-commutative geometry, giving almost zero cosmological constants $\Lambda_{1}$ and $\Lambda_{2}$ (see [1]).

At the early stage, the Universe was very hot, but then it began to cool down. Blackholes-monopoles (as bubbles of the vapour in the boiling water) began to disappear. The temperature dependent part of the energy density died away. In that case, only the vacuum energy will survive. Since this is a constant, the Universe expands exponentially, and an exponentially expanding Universe leads to the inflation (see reviews [52,53]). While the Universe was expanding exponentially, so it was cooling exponentially. This scenario was called supercooling in the false vacuum. When the temperature reached the critical value $T_{c}$, the Higgs mechanism of the SM created a new condensate $\phi_{m i n 1}$, and the vacuum became similar to a superconductor, in which the topological defects are magnetic vortices. The energy of black-holes is released as particles, which were created during the radiation era of the Universe, and all these particles (quarks, leptons, vector bosons) acquired their masses $m_{i}$ through the Yukawa coupling mechanism $Y_{f} \bar{\psi}_{f} \psi_{f} \phi$. Therefore, they acquired the Compton wavelength, $\lambda_{i}=\hbar / m_{i} c$. Then according to the Sidharth's theory of the cosmological constant, in the EW-vacuum we again have latticelike structures formed by bosons and fermions, and the lattice parameters " $l_{i}$ " are equal to the Compton wavelengths: $l_{i}=\lambda_{i}=\hbar / m_{i} c$.

\section{$7 \quad$ Stability of the EW-vacuum}

Here we emphasize that due to the energy conservation law, the vacuum density before the phase transition (for $T>T_{c}$ ) is equal to the vacuum density after the phase transition (for $T<T_{c}$ ), therefore we have:

$$
\rho_{v a c}(\text { at Planck scale })=\rho_{v a c}(\text { at EW scale }) \text {. }
$$

The analogous link between the Planck scale phase and EW phase was considered in the paper [50]. It was shown that the vacuum energy density (DE) is described by the different contributions to the Planck and EW scale phases. This difference is a result of the phase transition. However, the vacuum energy densities (DE) of both vacua are 
equal, and we have a link between gravitation and electromagnetism via the Dark Energy. According to the last equation (79), we see that if $\rho_{v a c}$ (at the Planck scale) is almost zero, then $\rho_{v a c}$ (at EW scale) also is almost zero, and we have a triumph of the Multiple Point Principle: we have two degenerate vacua with almost zero vacuum energy density. Almost zero cosmological constants are equal:

$$
\Lambda_{1}=\Lambda_{2} \approx 0
$$

Now we have obtained that the EW-vacuum, in which we live, is stable. The Planck scale vacuum cannot be negative: $V_{\text {eff }}\left(\min _{1}\right)=V_{\text {eff }}\left(\min _{2}\right)$.

\section{Conclusions}

1. In the present paper, we investigated the topological structure of the universal vacua. Different phase transitions, which were resulted during the expansion of the early Universe after the Planck era, produced the formation of the various kind of topological defects in vacua of the Universe. The aim of this investigation is the consideration of the hedgehog configurations as defects in the false vacuum. We have obtained a solution for a blackhole in the region which contains a global monopole in the framework of the $f(R)$ gravity, where $f(R)$ is a function of the Ricci scalar $R$. Here we have used the results of the Gravi-Weak unification (GWU) model. The gravitational field, isovector scalar $\Phi^{a}$ with $a=1,2,3$, produced by a spherically symmetric configuration in the scalar field theory, is pointing radially: $\Phi^{a}$ is parallel to $\hat{r}$ - the unit vector in the radial direction. In this GWU approach, we obtained a "hedgehog" solution (in Alexander Polyakov's terminology). We also showed that this is a black-hole solution, corresponding to a global monopole that has been "swallowed" by a black-hole.

2. In contrast to the previous theory [1-3], here we have taken into account the contribution of the magnetic field of hedgehogs.

3. We were based on the discovery that a cosmological constant of our Universe is extremely small, almost zero, and assumed a new law of Nature which was named as a Multiple Point Principle (MPP). The MPP postulates: There are two vacua in the SM with the same energy density, or cosmological constant, and both cosmological constants are zero, or approximately zero. We considered the existence of the following two degenerate vacua in the SM: a) the first Electroweak vacuum at $v_{1}=246 \mathrm{GeV}$, which is a "true" vacuum, and b) the second "false" vacuum at the Planck scale with VEV $v_{2} \sim 10^{18} \mathrm{GeV}$.

4. The bubble, which we refer to as "the false vacuum", is a de Sitter space with its constant expansion rate $H_{F}$. The initial radius of this bubble is close to the de Sitter horizon, which corresponds to the Universe radius. The space-time inside the bubble, which we refer to as "the true vacuum", has the geometry of an open FLRW universe.

5. By solving the gravitational field equations we estimated the black-hole-hedgehog's mass, radius $\delta$ and horizon radius $r_{h}$. They are: $M_{B H} \approx 3.65 \times 10^{18} \mathrm{GeV}, \delta \approx 6 \cdot 10^{-21}$ $\mathrm{GeV}^{-1}$ and $r_{h} \approx 1.14 \delta$. 
6. We estimated all parameters of the Gravi-Weak unification model, which gave the prediction of the Planck scale false vacuum VEV equal to $v=2 \sqrt{2} M_{P l}^{\text {red }} \approx 6.28 \times 10^{18}$ $\mathrm{GeV}$.

7. We have shown, that the Planck scale Universe vacuum is described by a nondifferentiable space-time: by a foam of black-holes, or by lattice-like structure, where sites are black-holes with the "hedgehog" monopoles inside them. This manifold is described by a non-commutative geometry, leading to a tiny value of cosmological constant $\Lambda \approx 0$.

8. Taking into account that the phase transition from the "false vacuum" to the "true vacuum" is a consequence of the electroweak spontaneous breakdown of symmetry $S U(2)_{L} \times U(1)_{Y} \rightarrow U(1)_{e l . m a g}$, we considered topological defects of EW-vacuum: the Abrikosov-Nielsen-Olesen closed magnetic vortices ("ANO strings") of the Abelian Higgs model and Sidharth's Compton phase objects. We showed that the "true vacuum" (EWvacuum) again is presented by the non-differentiable manifold with non-commutative geometry leading to an almost zero cosmological constant.

9. We considered that due to the energy conservation law, the vacuum energy density before the phase transition is equal to the vacuum energy density after the phase transition: $\rho_{\text {vac }}($ at Planck scale $)=\rho_{\text {vac }}($ at EW scale $)$. This result confirms the Multiple Point Principle: we have two degenerate vacua $v_{1}$ and $v_{2}$ with an almost zero vacuum energy density (cosmological constants). By this consideration we confirmed the vacuum stability of the EW-vacuum, in which we live. The Planck scale vacuum cannot be negative because of the exact equality $V_{\text {eff }}\left(\min _{1}\right)=V_{\text {eff }}\left(\min _{2}\right)$.

\section{Acknowledgments}

LVL greatly thanks to the B.M. Birla Science Centre (Hyderabad, India) and personally Prof. B.G. Sidharth, for hospitality, collaboration and financial support. HBN wishes to thank the Niels Bohr Institute for the status of professor emeritus and corresponding support. CRD is thankful to Prof. D.I. Kazakov for support.

\section{Appendix A. Parameters of the Gravi-Weak unifica- tion model}

At the first stage of the evolution (before the inflation), the Universe had the de Sitter spacetime - maximally symmetric Lorentzian manifold with a constant and positive background scalar curvature $R$. Then we have the following relations from the action (2):

1) The vacuum expectation value $v_{2}$ - the VEV of "the false vacuum" - is given by the de Sitter scalar curvature $R$ :

$$
v_{2}^{2}=\frac{R}{3}
$$


2) At the Planck scale the squared coupling constant of the weak interaction is:

$$
g_{2}^{2}=g_{\text {uni }} .
$$

The replacement:

$$
\frac{\Phi^{a}}{g_{2}} \rightarrow \Phi^{a}
$$

leads to the following GW-action:

$$
\left.S_{(G W)}=-\int_{\mathfrak{M}} d^{4} x \sqrt{-g}\left(\frac{R}{16}|\Phi|^{2}-\frac{3 g_{2}^{2}}{32}|\Phi|^{4}+\frac{1}{2} \mathcal{D}_{\mu} \Phi^{\dagger} \mathcal{D}^{\mu} \Phi+\frac{1}{4 g_{2}^{2}} F_{\mu \nu}^{i} F^{i \mu \nu}\right)+\text { grav. terms }\right) .
$$

Now considering the VEV of the false vacuum as $v=v_{2}$, we have:

$$
v^{2}=\frac{R}{3 g_{2}^{2}}
$$

The Einstein-Hilbert action of general relativity with the Einstein's cosmological constant $\Lambda_{E}$ is given by the following expression:

$$
S_{E H}=-\frac{1}{\kappa} \int d^{4} x \sqrt{-g}\left(\frac{R}{2}-\Lambda_{E}\right)
$$

3) The comparison of the Lagrangian $L_{E H}$ with the Lagrangian given by Eq. (A4) near the false vacuum $v$ leads to the following relation between the Newton's gravitational constant $G_{N}$ and reduced Planck mass:

$$
\left(M_{P l}^{r e d}\right)^{2}=\left(8 \pi G_{N}\right)^{-1}=\frac{1}{\kappa}=\frac{v^{2}}{8} .
$$

4) Then we have:

$$
v=2 \sqrt{2} M_{P l}^{r e d} \approx 6.28 \times 10^{18} \mathrm{GeV}
$$

and

$$
\Lambda_{E}=\frac{3 g_{2}^{2}}{4} v^{2}
$$

Eq. (A7) gives:

$$
\frac{1}{\kappa} \Lambda_{E}=\frac{3 g_{2}^{2}}{32} v^{4}
$$

The coupling constant $g_{2}$ is a bare coupling constant of the weak interaction, which coincides with a value of the constant $g_{2}$ at the Planck scale. Considering the renormalization group equation (RGE) for the $\mathrm{SU}(2)$ running constant $\alpha_{2}^{-1}(\mu)$, where $\alpha_{2}=g_{2}^{2} / 4 p i$ (see Refs. [34, 35]), we can carry out an extrapolation of this rate to the Planck scale, what leads to the following estimations:

$$
\alpha_{2}\left(M_{P l}\right) \sim \frac{1}{50}, \quad g_{\text {uni }}=g_{2}^{2}=4 \pi \alpha_{2}\left(M_{P l}\right) \approx 4 \pi \times 0.02 \approx 0.25
$$




\section{Appendix A. Global monopole}

A global monopole is described by the part $L_{h}$ of the Lagrangian $L_{(G W)}$ given by the action (A4), which contains the $S U(2)$-triplet Higgs field $\Phi^{a}$, VEV of the second vacuum $v_{2}=v$ and cosmological constant $\Lambda=\Lambda_{E}$ :

$$
\begin{gathered}
L_{h}=-\frac{R}{16}|\Phi|^{2}+\frac{3 g_{2}^{2}}{32}|\Phi|^{4}-\frac{1}{2} \partial_{\mu} \Phi^{a} \partial^{\mu} \Phi^{a}+\Lambda_{E} \\
=-\frac{1}{2} \partial_{\mu} \Phi^{a} \partial^{\mu} \Phi^{a}+\frac{\lambda}{4}\left(|\Phi|^{2}-v^{2}\right)^{2}+\frac{\Lambda_{E}}{\kappa}-\frac{\lambda}{4} v^{4}=-\frac{1}{2} \partial_{\mu} \Phi^{a} \partial^{\mu} \Phi^{a}+\frac{\lambda}{4}\left(|\Phi|^{2}-v^{2}\right)^{2} .
\end{gathered}
$$

Here we have:

$$
\lambda=\frac{3 g_{2}^{2}}{8}
$$

Substituting in Eq. (A13) the value $g_{2}^{2} \approx 0.25$ given by Eq. (A11), we obtain:

$$
\lambda \approx \frac{3}{32}
$$

Eq. (A9) gives:

$$
\frac{\Lambda_{E}}{\kappa}=\frac{3 g_{2}^{2}}{32} v^{4}=\frac{\lambda}{4} v^{4},
$$

and in Eq. (A12) we have the compensation of the Einstein's cosmological term. Then

$$
L_{h}=-\frac{1}{2} \partial_{\mu} \Phi^{a} \partial^{\mu} \Phi^{a}+V(\Phi)
$$

where the Higgs potential is:

$$
V(\Phi)=\frac{\lambda}{4}\left(|\Phi|^{2}-v^{2}\right)^{2}
$$

This potential has a minimum at $\langle|\Phi|\rangle_{\min }=v$, in which it vanishes:

$$
V\left(|\Phi|_{\min }^{2}\right)=V^{\prime}\left(|\Phi|_{\min }^{2}\right)=0
$$

in agreement with the MPP conditions (19) and (20).

\section{References}

[1] B.G. Sidharth, C.R. Das, L.V. Laperashvili, and H.B. Nielsen, Int. J. Mod. Phys. D27, 1850022 (2018), arXiv:1703.05594v2[hep-ph].

[2] C.R. Das, L.V. Laperashvili, B.G. Sidharth, and H.B. Nielsen, Black-holes-hedgehogs in the false vacuum and a new physics beyond the Standard Model, J. Phys. Conf. Ser. 934, 012043 (2017).

[3] B.G. Sidharth, C.R. Das, C.D. Froggatt, H.B. Nielsen, and Larisa Laperashvili, Degenerate Vacua of the Universe and What Comes Beyond the Standard Model, New Advances in Physics, Vol.12, No 1, pp.1-23, Research Science Press, ed. B.G. Sidharth, June 2018, India, ISSN:0974-3553; arXiv:1801.06979 [hep-ph]. 
[4] G. t’Hooft, Nucl. Phys. B79, 276 (1974).

[5] A.M. Polyakov, Pisma Zh. Eksp. Teor. Fiz. 20, 43 (1974) [JETP Lett. 20, 194 (1974)].

[6] M.S. Volkov, D.V. Gal'tsov, Gravitating non-Abelian solitons and black holes with Yang-Mills fields, Phys. Rept. 319, 1-83 (1999), e-print: hep-th/9810070.

[7] C. R. Das, L. V. Laperashvili and A. Tureanu, Int. J. Mod. Phys. A 28, 1350085 (2013), arXiv:1304.3069.

[8] C. D. Froggatt, C. R. Das, L. V. Laperashvili, H. B. Nielsen and A. Tureanu, arXiv:1311.4413.

[9] L. V. Laperashvili, H. B. Nielsen and A. Tureanu, Int. J. Mod. Phys. A 30, 1550044 (2015), arXiv:1411.6456.

[10] C. R. Das, L. V. Laperashvili, H. B. Nielsen, A. Tureanu and C. D. Froggatt, Phys. Atom. Nucl. 78, 440 (2015) [Yad. Fiz. 78, 471 (2015)].

[11] A. Garrett Lisi, L. Smolin and S. Speziale, J. Phys. A 43, 445401 (2010), arXiv:1004.4866.

[12] D. L. Bennett, L. V. Laperashvili, H. B. Nielsen and A. Tureanu, Int. J. Mod. Phys. A 28, 1350035 (2013), arXiv:1206.3497.

[13] S.M. Carrol, V. Duvvuri, M. Troden and M.S. Turner, Phys. Rev. D70, 043528 (2004).

[14] S. Fay, R. Tavakol and S. Tsujikawa, Phys. Rev. D74, 063509 (2007).

[15] S. Nojiri and S.D. Odintsov, Phys. Lett. B657, 238 (2007).

[16] P. Chen, Mod. Phys. Lett. A 25 (2010) 2795; arXiv:1002.4275.

[17] S. D. Odintsov and V. K. Oikonomou, arXiv:1508.07488,

[18] Particle Data Group (K.A. Olive et al.), 2014, 1676 pp. Published in Chin. Phys. C38, 090001 (2014).

[19] B.G. Sidharth, Proc. of the 8th Marcell Grossmann Meeting on General Relativity, Jerusalem, May 1997, ed. T. Piran, (World Scientic, Singapore), pp. 476-479 (1997).

[20] B.G. Sidharth, Int. J. Mod. Phys. A13, 2599 (1998), arXiv:quant-ph/9808031.

[21] S. Perlmutter, B. Schmidt and A. Riess, Nature 391, 51 (1998).

[22] D.L. Bennett and H.B. Nielsen, Int. J. Mod. Phys. A9, 5155 (1994), arXiv:hep-ph/9311321.

[23] D.L. Bennett, C.D. Froggatt and H.B. Nielsen, in Proceedings of the 27th International Conference on High Energy Physics, Glasgow, Scotland, 1994, eds. P. Bussey and I. Knowles (IOP Publishing Ltd., 1995), p. 557.

[24] C.D. Froggatt and H.B. Nielsen, Phys. Lett. B368, 96 (1996), arXiv:hep-ph/9511371.

[25] L.V. Laperashvili, Phys. Atom. Nucl. 57, 471 (1994) [Yad.Fiz. 57, 501 (1994)]. 
[26] E. J. Copeland, M. Sami and S. Tsujikawa, Int. J. Mod. Phys. D 15 (2006) 1753, 3 arXiv:hep-th/0603057.

[27] M. Barriola and A. Vilenkin, Phys. Rev. Lett. 63, 341 (1989).

[28] R. Basu, A. H. Guth and A. Vilenkin, Phys. Rev. D44, 340 (1991).

[29] A. Vilenkin, JCAP 1405, 005 (2014), arXiv:1312.0682,

[30] J. Garriga, A. Vilenkin and Jun Zhang, JCAP 1602, 064 (2016), arXiv:1512.01819.

[31] L.F. Abbott and S. Deser, Nucl. Phys. B195, 76 (1982).

[32] L.H. Ford, Phys. Rev. D31, 710 (1985).

[33] I. Antoniadis, J. Iliopoupos, and T.N. Tomaras, Phys. Rev. Lett. 56, 1319 (1986).

[34] D.L. Bennett, L.V. Laperashvili and H.B. Nielsen, Finestructure constants at the Planck scale from multiple point principle, in: Proceedings of the 10th Workshop on What Comes Beyond the Standard Model, Bled, Slovenia, 17-27 September, 2007, eds. M. Breskvar et al. (Bled Workshops in Physics, Vol. 8 no. 2, DMFA-Zaloznistvo, Ljubljana, 2007), arXiv:0711.4681.

[35] D.L. Bennett, L.V. Laperashvili and H.B. Nielsen, Relation between the structure constants at the Planck scale from multiple point principle, in: Proceedings of the 9th Workshop on What Comes Beyond the Standard Model, Bled, Slovenia, 16-26 September, 2006, eds. M. Breskvar et al. (Bled Workshops in Physics, Vol. 7, no. 2, DMFA-Zaloznistvo, Ljubljana, 2006), arXiv:hep-ph/0612250.

[36] E.I. Guendelman and A. Rabinowitz, Phys. Rev. D44, 3152 (1991).

[37] F.B. Lustosa, M.E.X. Guimaraes, C.N. Ferreira and J.L. Neto, Thermodynamical Analysis of a Black Hole with a Global Monopole Within a Class of a $f(R)$ Gravity, arXiv:1510.08176.

[38] O. Delice, JHEP 0311, 058 (2003), arXiv:gr-qc/0307099.

[39] Xin Shi and Xin-zhou Li, The gravitational field of a global monopole, arXiv:0903.3085.

[40] T. R. P. Caramês, E. R. Bezerra de Mello, J. Pessoa and M. E. X. Guimarães. On the motion of a test particle around a global monopole in a modified gravity, arXiv:1111.1856.

[41] R. Bartnik and J. McKinnon. Particlelike solutions of the Einstein Yang-Mills equations. Phys.Rev.Lett., 61, 141-144, 1988.

[42] B.G. Sidharth, The Thermodynamic Universe: Exploring the limits of physics (World Scientific, Singapore, 2008).

[43] C. Rovelli, Quantum Gravity, Cambridge Monographs on Mathematical Physics, 2007.

[44] H.S. Snyder, Phys. Rev. 72, 68 (1947). 
[45] B.G. Sidharth, The Chaotic Universe: From the Planck to the Hubble Scale (Nova Science, New York, 2001).

[46] Ya.B. Zeldovich, JETP Lett. 6, 316 (1967).

[47] S. Weinberg, Phys. Rev. Lett. 43, 1566 (1979).

[48] A.A. Abrikosov, Soviet JETP 32, 1442 (1957).

[49] H.B. Nielsen and P. Olesen, Nucl. Phys. B61, 45 (1973).

[50] B.G. Sidharth, A. Das and A.D. Roy, Int. J. Theor. Phys. 55, 801 (2016).

[51] B.G. Sidharth, Nuovo. Cim. B116, 735 (2001), arXiv:physics/0106051.

[52] A.D. Linde, Rep. Prog. Phys. 42, 389(1979).

[53] A.D. Linde, Inflation and Quantum Cosmology (Academic Press, Boston, 1990). 